\title{
Cultura y públicos en Uruguay: un estudio sobre el Ballet Nacional del Sodre
}

\author{
Culture and audiences in Uruguay: a study of the National Ballet \\ company of the Sodre (Official Service of Dissemination, \\ Representations and Shows)
}

\section{Diego Gómez Anchen,I Mariana Paredes, II Germán Silveira, III Julián SuquilvideIV y Danilo Urbanavicius ${ }^{\mathrm{V}}$}

I. Psicólogo, Universidad de la República. Docente y coordinador académico de la Tecnicatura en Gestión Cultural, Facultad de la Cultura, Universidad CLAEH. Coordinador ejecutivo en Auditorio Nacional del Sodre, 2012-2017. \dgomez@claeh.edu.uy.https://orcid.org/0000-0001-9403-9755

II. Licenciada en Sociología, Universidad de la República. Especialización en Análisis Demográfico para el Desarrollo, Centro Latinoamericano de Demografía, Chile. Doctora, Universidad Autónoma de Barcelona. Programa de Población y CIEN - Espacio Interdisciplinario, Facultad de Ciencias Sociales, UDELAR. Docente de la Facultad de la Cultura, Universidad CLAEH. $₫$ mparedes@claeh.edu.uy. https://orcid.org/0000-0002-2323-6097

III. Doctor en Estudios Transculturales de la Universidad Jean Moulin Lyon 3. Docente de la Facultad de la Cultura, Universidad CLAEH, y de la Maestría en Comunicación, Énfasis en Recepción y Cultura, Universidad Católica del Uruguay. Investigador nivel iniciación del Sistema Nacional de Investigadores ANII. \gsilveira72@gmail.com. https://orcid.org/0000-0001-9654-0594

IV. Magíster en Educación. Docente de Metodología de la Investigación y tutor de tesis de posgrado, Universidad CLAEH. Consultor en investigaciones sociales. $₫$ julian280846@gmail.com.

V. Técnico en Gestión Cultural, Facultad de la Cultura, Universidad CLAEH. Comunicador social. Desde 2009 trabaja en gestión de artes escénicas. Ha gestionado proyectos culturales públicos y privados. Asistente del Decanato de la Facultad de la Cultura, Universidad CLAEH. \durbanavicius@claeh.edu.uy.

\section{Resumen}

Este trabajo presenta los avances de una investigación sobre públicos del Ballet Nacional del Sodre realizada por la Facultad de la Cultura. A través de casi un siglo de trayectoria, la principal compañía de danza clásica en Uruguay, de carácter estatal, ha sufrido importantes transformaciones. La llegada del reconocido bailarín Julio Bocca a la dirección artística, en 2010, llevó a un cambio en el modelo de gestión de la compañía y a una nueva configuración del público. El estudio de públicos del Ballet se propone dimensionar sus características, su perfil sociodemográfico, sus opiniones, valoraciones y actitudes sobre el espectáculo, sus hábitos de concurrencia y sus expectativas. Los métodos 
utilizados combinan un enfoque cuantitativo, a través de la realización de una encuesta, y cualitativo, mediante grupos de discusión y observación participante. Los resultados que se presentan aquí forman parte de la primera etapa de esa investigación de públicos del Ballet, un trabajo que puede ser un aporte para el diseño, la formulación y la evaluación de las políticas culturales en ese ámbito.

Palabras clave: ballet, Uruguay, estudios culturales, usuario cultural.

\begin{abstract}
This work presents the advances of an investigation on audiences of the Ballet Nacional del SODRE (National Ballet Company of the Official Service of Dissemination, Representations and Shows), the main classical dance company in Uruguay. This state-run institution has undergone important transformations through almost a century of existence, one of which has taken place in recent years, when the renowned dancer Julio Bocca takes over as artistic director. This led to a change in the company's management and to an expansion towards a wider and massive audience. In this context it is considered relevant to carry out a research on Ballet audiences to assess their characteristics, their socio-demographic profile, their opinions, ratings and attitudes towards the show, their attendance habits and their expectations. The methods used combine a quantitative approach - through a survey - and a qualitative one - through focus groups and participant observation. Preliminary results at this first stage of the investigation provide input for future research and can be interpreted as a contribution to the design, formulation and evaluation of cultural policies in this filed.
\end{abstract}

Key words: ballet, Uruguay, cultural studies, cultural users

\title{
Presentación
}

En Uruguay hay público para el ballet. Las 50 funciones por año del Ballet Nacional del Sodre (BNS) y los más de 600.000 espectadores que asistieron entre 2010 y 2017 a sus espectáculos ${ }^{1}$ son la evidencia empírica de este nuevo fenómeno cultural. A partir de una serie de transformaciones que sufrieron las antiguas estructuras asociadas al BNS, el público volvió a ubicarlo en un lugar de preferencia entre las prácticas culturales. Desde la infraestructura que ofrece, tanto para los bailarines como para el público, el nuevo Auditorio Nacional, reinaugurado en noviembre de 2009 en Montevideo, hasta el modelo de gestión que llevó adelante el maestro Julio Bocca desde que asumió como director artístico, en junio de 2010, diversos factores dieron impulso a una nueva etapa 
de la compañía. Al mismo tiempo, esas transformaciones llevaron a una reconfiguración del público más clásico con el que generalmente se asocia a los espectáculos de ballet.

Para dar cuenta de la dimensión de estos cambios y para caracterizar al público actual del Ballet, la Facultad de la Cultura de la Universidad CLAEH, en convenio con el Ballet Nacional del Sodre, pone en marcha el proyecto «Estudio de públicos del BNS» en junio de 2017. Se presentan aquí los principales avances de esta investigación —aún en curso- con el fin de proporcionar un panorama descriptivo del público que asiste al BNS: su perfil sociodemográfico, sus preferencias culturales, las motivaciones para asistir al ballet, así como la valoración de la compañía en general y del espectáculo que presenciaron en particular. El análisis que aquí se ofrece tiene carácter preliminar, en el entendido de que una versión final de las conclusiones podrá presentarse al culminar la investigación.

\section{Estudio de públicos del BNS}

El Cuerpo de Baile del Sodre se creó en agosto de 1935 como un organismo artístico público encargado de desarrollar el ballet clásico profesional en Uruguay. Su primer director fue el maestro Alberto Pouyanne, y durante las primeras décadas la compañía recibió la influencia de las escuelas francesa, rusa y norteamericana. La llegada de compañías, maestros y bailarines procedentes de una Europa convulsionada por el nazismo y las persecuciones a intelectuales y artistas situó a Montevideo dentro del circuito de giras que se realizaban en América, y la cercanía del Colón de Buenos Aires favorecía la visita de sus espectáculos.

La compañía nacional tuvo una época dorada en los años sesenta, como resultado de la llegada de los bailarines argentinos Margaret Graham, Tito Barbón y Eduardo Ramírez desde el Ballet de La Plata en 1957, impulsada por el reconocido maestro polaco Yurek Shabelevsky (que bailó durante varias temporadas en el Teatro Colón de Buenos Aires entre 1937 y 1947) (Craine y Mackrell, 2010, p. 406), y de la conformación de un elenco uruguayo de grandes figuras que integraron el cuerpo de baile en diferentes momentos (Olga Banegas, Flor de María Rodríguez, Micha Dimitrievich, Alfredo Corvino, Marina Korolkov, Sara Nieto y muchos otros). En 1962 se llegaron a realizar 57 funciones, un récord absoluto en la historia de la compañía (Campos, 2014, p. 6). Graham y Barbón crearon, además, la Escuela Nacional de Danza, para fortalecer ese tipo de formación por entonces ausente en el país.

Años más tarde, el 18 de setiembre de 1971, un incendio destruyó el edificio del Estudio Auditorio y la compañía de ballet quedó sin la infraestructura adecuada para cumplir su tarea. Durante mucho tiempo la sala Brunet (hoy sala Nelly Goitiño), en 18 
de Julio y Andes, suplió precariamente esa falta, pero el ballet perdió brillo y dejó de ocupar un lugar en la consideración del público entre la oferta cultural.

Esta investigación tiene como objetivo general hacer un estudio sobre el público contemporáneo que asiste a los espectáculos del Ballet Nacional del Sodre en Montevideo. En una primera etapa se busca analizarlo en sus características sociodemográficas, preferencias, motivaciones y opiniones sobre el espectáculo y la compañía. En una segunda etapa $-\mathrm{y}$ a partir de insumos recogidos en la primera- se buscará indagar en las motivaciones, representaciones y apropiación simbólica que el público establece en su relación con el ballet como práctica cultural.

El artículo repasará algunos resultados preliminares de la primera etapa del estudio, en el análisis de dos obras (Gala de Ballet VI y Romeo y Julieta) de la temporada 2017. En la Facultad de la Cultura, creemos que la experiencia del ballet es digna de ser observada. El BNS se encuentra, además, en una coyuntura particular: tiene una autonomía y una fuerza más allá de la institución que lo cobija, y el apoyo del público resulta por momentos sorprendente. En este sentido, como expresan Wortman y Bayardo, el estudio de los consumos culturales «contribuye a la comprensión de los procesos sociales y políticos, la conformación de subjetividades y la construcción de identidades colectivas», así como «aporta al análisis de las políticas culturales, tanto en lo referente a su evaluación como en perspectivas para su diseño e implementación» (Wortman y Bayardo, 2012, p. 12).

Las cifras de asistencia al BNS son elocuentes en cuanto al crecimiento y la aceptación que gradualmente la compañía fue adquiriendo en su etapa de refundación, a partir de la llegada de Julio Bocca a la dirección artística, en 2010. En agosto de ese año, con la puesta en escena de Giselle - primera obra de esta nueva etapa-, el BNS alcanzaba unos 20.500 espectadores. Cinco años más tarde, cuando se reponía Giselle, se consagraba como la obra más exitosa de la compañía, con 25.500 espectadores (Auditorio Nacional del Sodre, s/f). Se pasó de 49.000 espectadores en 2010 a más de 107.000 en 2017. Estas cifras, que se presentan en el siguiente cuadro, muestran que se ha logrado operar un cambio de magnitud en el consumo cultural relacionado con esa danza. En promedio, en el período comprendido entre 2010 y 2017, el BNS ofreció 53 funciones por año y a cada una asistieron unas 1700 personas. Esto indica que las funciones estuvieron al límite de la capacidad de la sala principal Eduardo Fabini, que cuenta con un aforo máximo de 1885 espectadores. 
Cuadro 1. Cantidad de funciones ordinarias y de espectadores por año

\begin{tabular}{|l|c|c|}
\hline Año & Funciones & Espectadores \\
\hline 2010 & 30 & 49.115 \\
\hline 2011 & 40 & 64.073 \\
\hline 2012 & 40 & 65.760 \\
\hline 2013 & 45 & 73.607 \\
\hline 2014 & 49 & 98.026 \\
\hline 2015 & 48 & 90.024 \\
\hline 2016 & 52 & 82.848 \\
\hline 2017 & 66 & 107.952 \\
\hline Total & $\mathbf{3 7 0}$ & $\mathbf{6 3 1 . 4 0 5}$ \\
\hline
\end{tabular}

En 2017 Montevideo contaba con una población de 1.263.705 habitantes. ${ }^{2}$ Por lo tanto, un $8,4 \%$ de los montevideanos asistió ese año a los espectáculos del BNS. Para pensar este fenómeno desde una perspectiva más amplia, habría que estudiarlo comparativamente con otras ciudades capitales, algo que escapa a los límites de este artículo, pero que puede ser un próximo paso para entender algunas de las políticas culturales que se desarrollan simultáneamente en la región.

Los datos que surgen del Tercer informe nacional sobre consumo y comportamiento cultural, correspondientes a 2014, confirman esta tendencia en la consideración del público hacia el ballet, en todo el territorio nacional. En 2002, cuando se realizó el Primer informe, un $15 \%$ de los encuestados declaró haber asistido a algún espectáculo de danza en el último año. La expresión espectáculo de danza comprendía el folklore, el ballet, la danza moderna, el tango, la salsa y otras (Achugar, Rapetti, Dominzain y Radakovich, 2002, p. 89). En 2014 esa cifra aumentó en 11 puntos porcentuales: alcanzó el 26\%. En cuanto a la danza más vista, en 2009 el folklore se ubicaba en el primer lugar, con un $50,5 \%$. Algunos años más tarde, en 2014 , el folklore fue mencionado por un $33,4 \%$, lo viembre de 2013. Actualización de datos al 2017 basada en cálculos propios por tasa de crecimiento poblacional. 
que indica una caída considerable en las preferencias. En cambio, el ballet es la danza que más crecimiento experimentó: mientras que en 2009 mencionaba haber visto ballet un 26,3\% de la muestra, en 2014 esa proporción ascendió al 45\% (Dominzain, Radakovich, Duarte y Castelli Rodríguez y, 2014, p. 140-141). El ballet se ubica así como el espectáculo de danza más visto por el público en Uruguay.

Ahora bien, algunas preguntas comienzan a surgir a partir de estos primeros datos. ¿Cómo es ese público del ballet? ¿Dónde vive? ¿Cómo son sus características por sexo, edad y nivel educativo? ¿Cuáles son las prácticas culturales de este público y las motivaciones para asistir al ballet? ¿Qué piensa del Ballet Nacional del Sodre? ¿Cuánto peso tuvo la figura de Julio Bocca en su decisión de acercarse al BNS? ¿Qué porcentaje de espectadores asiste por primera vez al ballet? ¿Sigue perteneciendo el público del ballet a una elite social?

El estudio del público o, mejor, de los públicos del Ballet Nacional se propone indagar en estas cuestiones que contribuyen a comprender la relación entre los espectadores y los espectáculos de ballet. El estudio de públicos como campo específico de conocimiento procura entonces reconstruir procesos de involucramiento en el circuito de producción y recepción cultural y artístico. Busca desentrañar la naturaleza de ese involucramiento, los móviles, las características del público, las formas de reconstrucción de la experiencia. Puede ser de buen auxilio a productores, programadores, comunicadores, gestores culturales o quienes tengan a su cargo la definición de políticas de corte más general.

\section{Algunos antecedentes de estudios sobre públicos de la cultura}

Publicado originalmente en Francia en 1969, El amor del arte (2003) es el título de una investigación dirigida por Pierre Bourdieu y Alain Darbel sobre el público de los museos en Europa. Este estudio de referencia en el ámbito de la sociología de las prácticas culturales tenía como objetivo indagar en el conjunto de causas y razones que permitieran explicar y comprender la visita a los museos, para lo que examinaba las condiciones generales de recepción de una obra (características sociales y escolares de ese público, actitudes con respecto al museo que visitaba y preferencias artísticas). La concepción de Bourdieu del campo social como un espacio organizado según un sistema de posiciones jerarquizadas en función de la situación socioeconómica de las diferentes clases ya se hacía presente en este trabajo. Así, El amor del arte reveló una relación entre las tasas de frecuentación de los museos y las categorías sociales y profesionales: el número de visitas a un museo es mayor cuanto más alto es el estatus social. Del mismo modo, las 
razones por las cuales los visitantes van al museo, así como los comportamientos frente a una obra de arte, difieren según el nivel social. La competencia estética, adquirida a través de una familiaridad con las obras de arte, constituirá otro de los factores de distinción en las prácticas culturales que el estudio pone de relieve.

Conociendo a nuestros visitantes (Ministerio de Cultura de España. Laboratorio Permanente de Público de Museos, 2011) es una investigación más reciente, elaborada por el Ministerio de Cultura español en colaboración con la Universidad Complutense de Madrid entre 2007 y 2009, con el fin de conocer características, hábitos, motivaciones, opiniones y valoraciones del público en doce museos estatales. El marco teórico adoptado para este trabajo sigue el modelo de experiencia interactiva propuesto por Dierking y Falk (1992) en The Museum Experience (citado por Ministerio de Cultura de España. Laboratorio Permanente de Público de Museos, 2011), donde se plantea repensar los museos desde la perspectiva del visitante.

Según estos autores, durante la visita a un museo interactúan tres tipos de contextos: personal, social y físico. El contexto personal es entendido como las características sociodemográficas, los intereses, las motivaciones y las inquietudes de los visitantes. El contexto social, que influiría significativamente en la experiencia museística, tiene en cuenta a las personas con las se realiza la visita, así como las interacciones con otros visitantes y con el personal del museo. El contexto físico es definido por estos autores como los aspectos arquitectónicos, objetos y artefactos que contiene el museo. Centrada en la interacción de estos tres factores, la investigación desarrolla un extenso análisis estadístico de la composición del público de los museos, la frecuencia, los motivos, la planificación de la visita, así como la valoración de la experiencia, a partir de un cuestionario entregado por un entrevistador y completado por el visitante, aplicado en una muestra de 12.000 visitantes en el período de un año.

Aunque no muy numerosos, los estudios de públicos de cine han adquirido cierta notoriedad en los últimos años, sobre todo a partir del interés que en algunos ámbitos académicos y también estatales se les ha otorgado a las audiencias de los medios masivos. Las preguntas en torno a las posibilidades de un cine nacional en un contexto de globalización y la relación con las nuevas identidades culturales de los públicos hacen de estos estudios - de corte cualitativo algunos y cuantitativo otros - una referencia pertinente para el caso que nos ocupa (BNS).

Así, en De la americanización a la globalización de los espectadores, la investigadora argentina Ana Wortman (2015) da cuenta de las transformaciones que ha ido sufriendo el público de cine desde la mitad del siglo xx hasta nuestros días. Wortman señala la importancia que hasta los años setenta tuvieron las salas de cine como «ámbito de consumo cultural central en las actividades de esparcimiento de las emblemáticas clases medias argentinas» (Wortman, 2015, p. 115). Ese sector social de clases medias que se caracterizaba entonces por «un gusto cultural diverso, universal, fuertemente 
informado y ávido de lo nuevo» (p. 115) tenía un alto grado de receptividad hacia las experimentaciones del llamado cine de autor que impulsaron las nuevas olas cinematográficas, lo que lleva a la autora a plantear: «Si pretendemos pensar en alguna política cultural de fomento y configuración de públicos para los nuevos cines nacionales, deberemos considerar las modificaciones de nuestras sociedades, cómo se insertan ellas en los contextos internacionales, los nuevos estilos de vida y los procesos históricos culturales que las constituyen» (p. 116).

Wortman y De Angelis (2017) exploraron los públicos de un evento cinematográfico en particular, el Buenos Aires Festival Internacional de Cine Independiente (BAFICI), con un enfoque cuantitativo, a partir de una encuesta de 300 casos al público de los distintos espacios donde se proyectaron películas durante los 15 días del festival. El dinero es lo de menos. A propósito del público del Festival BAFICI es un estudio que se apoya, en gran medida, en el marco teórico propuesto por la sociología de la cultura de Bourdieu, al dar cuenta de la importancia que tiene el capital cultural en el acceso de los públicos a los consumos culturales. En este sentido, la numerosa concurrencia del BAFICI (alrededor de 400.000 personas) viene a reafirmar, según esta investigación, que en el caso del consumo de películas del cine independiente «el acceso no está determinado por el costo (el precio de la entrada es inferior al ticket habitual al cine), sino por la exclusiva posesión de un capital cultural que parece en la actualidad estar vinculado en gran medida a la pertenencia a cierto ámbito económico» (Wortman y De Angelis, 2017).

La Facultad de la Cultura de la Universidad CLAEH también llevó adelante, entre 2009 y 2010, un estudio sobre el público cinematográfico en Uruguay, en el marco del proyecto Efecto Cine, en las localidades del país donde tuvieron lugar las proyecciones de cine itinerante de esa iniciativa (Centro Latinoamericano de Economía Humana [CLAEH], 2010). Desde sus inicios en 2008 hasta 2010, Efecto Cine había realizado unas 300 funciones y alcanzado a más de 250.000 espectadores en lugares muy diferentes del país (Aceguá, Río Branco, Dolores, Carmelo, San Gregorio de Polanco, Cardal, entre otras muchas localidades). A partir de los objetivos, que proponían medir el impacto del proyecto, se diseñó una investigación que articuló los enfoques cuantitativo (cuestionarios autoadministrados) y cualitativo (observación participante). De esta manera se logró elaborar un estudio que contemplara las características básicas de la población encuestada, algunas características vinculadas al consumo de cine, las preferencias de dicho consumo, así como el contexto de recepción de las exhibiciones.

Los estudios del público de la danza son aún muy escasos. Algunos trabajos recientes sobre el tema aportan, sin embargo, una mirada nueva e interesante sobre el objeto. Un estudio llevado a cabo en Costa Rica entre agosto de 2015 y junio de 2016, sobre el público de la danza contemporánea en el área metropolitana de San José, indaga en la cuestión desde una perspectiva cualitativa (Programa Nacional para el Desarrollo de las Artes Escénicas [PROARTES], 2016). Para ello combina sondeos al público asistente 
a la danza contemporánea, sondeos al público asistente a otras actividades culturales, grupos de discusión, observación, entrevistas en profundidad y análisis de fanpages en las redes sociales. La investigación, que se enmarca en el Programa Nacional para el desarrollo de las Artes Escénicas (PROARTES) 2015, se planteó explorar las tipologías que componen el público actual de la danza en aquel territorio. De esta manera, se trabajó con el perfil psicográfico del público para clasificarlo según sus actitudes, estilos de vida y personalidad, siguiendo el modelo de la teoría de Myers-Briggs. Entre las conclusiones de la investigación se destaca que la diferencia más importante encontrada entre quienes asisten frecuentemente a espectáculos de danza y quienes lo hacen poco o nada es que estos últimos perciben la danza como un esfuerzo cognitivo considerable, al tiempo que no establecen ningún tipo de conexión emocional con el género.

Por último, haremos referencia al trabajo realizado en Francia por Daniel Urrutiaguer (2014) sobre Las representaciones de los públicos en la danza contemporánea. Si bien en Francia la danza tuvo un impulso estatal considerable durante los años ochenta, con la creación de Centros Coreográficos Nacionales (CCN), no fue hasta los años dos mil que los estudios sobre el público — dice el autor- empezaron a ser indispensables, por la alta competencia que representan para estos centros las compañías de danza. De este modo, desde 2005 el Centro Nacional de la Danza (CND), creado en 1998, realiza estudios sobre el público con el foco en la composición sociodemográfica, de los que resulta que casi la mitad del público está en París. Pero, más allá de los datos cuantitativos, el autor se detiene en una variable que resulta muy significativa en el mundo de la danza para la atracción de públicos por las estructuras disponibles (establecimientos culturales, compañías, teatros), que es la reputación del coreógrafo. En este sentido, Urrutiaguer señala: «Los lazos de fidelidad de los espectadores con un coreógrafo parecen mucho más estrechos que los lazos que se puedan establecer con un director de teatro» (2014, p. 34). En consecuencia, los responsables de los establecimientos culturales están obligados a proteger su reputación corporativa y no descuidar ese aspecto si quieren ganar el favor del público.

\section{Abordaje metodológico}

El estudio de las políticas culturales como campo interdisciplinario suele reconocer una variedad de abordajes. En este caso, nos interesamos por los receptores de esas políticas culturales; esto es, por lo que la sociología de la cultura entiende como prácticas culturales. Por práctica cultural nos referimos, siguiendo a Coulangeon, al «conjunto de actividades de consumo o participación relacionados con la vida intelectual y artística que, a su vez, representan disposiciones estéticas y participan en la definición de estilos de 
vida: lectura, frecuentación de lugares de cultura (teatros, museos, salas de cine, conciertos), uso de los medios audiovisuales, así como prácticas amateurs» (Coulangeon, 2005, p. 4). ${ }^{3}$ La asistencia a los espectáculos de ballet se inscribe así en el conjunto de prácticas culturales seleccionadas por los individuos en su tiempo libre.

La investigación que desarrollamos reconoce dos grandes etapas. La primera tiene un enfoque de corte cuantitativo, con recopilación de datos a través de la técnica de encuesta. La segunda, de corte cualitativo, requiere la construcción teórica - mediante técnicas cualitativas de grupos de discusión y de observación participante- de los significados y representaciones que estos públicos les otorgan a sus prácticas. El presente artículo se centra en los resultados de la primera etapa, provenientes del trabajo de campo desarrollado en dos obras de la temporada 2017: Gala de Ballet VI y Romeo y Julieta.

Con el fin de recabar datos sobre las características sociodemográficas del público que asiste al ballet, así como de tener una primera aproximación a las opiniones sobre los espectáculos, la técnica aplicada con enfoque cuantitativo consistió en la distribución aleatoria de un cuestionario autoadministrado en los cinco pisos de la sala (platea baja, platea alta, tertulia, galería baja y galería alta). Este formato implicó que el cuestionario fuera completado por los espectadores a la salida de los espectáculos que tuvieron lugar en la Sala Fabini del Auditorio Nacional del Sodre. Los encuestadores estaban presentes durante el llenado del cuestionario para prestar asesoramiento si se planteaban dudas, y eventualmente completarlos a solicitud de los encuestados. Se diseñó un muestreo aleatorio por estratos en los cincos pisos de la sala. Las encuestas se aplicaron durante tres funciones de la Gala de Ballet VI (julio-agosto 2017), en las que se obtuvieron 286 formularios, y cinco funciones de Romeo y Julieta (setiembre-octubre 2017), que proporcionaron 309 formularios. En ambos casos el margen de error se sitúa en +/-5\%, con un nivel de confianza del $95 \%$.

Se elaboró un cuestionario estructurado que contenía los siguientes temas:

1. Características sociodemográficas del espectador.

2. Motivos y características de su visita.

3. Opiniones acerca de la función.

4. Opiniones acerca del ballet en general. 


\section{Características de la realización del trabajo de campo}

La Gala de Ballet VI ${ }^{4}$ se exhibió desde el 27 de julio hasta el 6 de agosto de 2017. La encuesta fue realizada los días martes $1 .^{\circ}$, viernes 4 y domingo 6 de agosto. Se aplicaron 286 formularios. La obra Romeo y Julieta se exhibió desde el 20 de setiembre hasta el 4 de octubre de 2017. La encuesta fue realizada los días martes 26, viernes 29, sábado 30 de setiembre, domingo $1 .^{\circ}$ y miércoles 4 de octubre. Se aplicaron en promedio 65 formularios por día y en total se obtuvieron 309. Dada la estructura de la sala principal del Auditorio Nacional del Sodre, se procuró distribuir las encuestas en todos los pisos, para incluir la heterogeneidad del público asistente en la composición de la muestra.

\section{Algunos resultados preliminares ${ }^{5}$}

\section{Características sociodemográficas del público}

De las encuestas aplicadas en los dos primeros espectáculos de la investigación ${ }^{6}$ surge que el público que asiste al BNS es predominantemente femenino, de edades adultas ${ }^{7} \mathrm{y}$ con un acentuado perfil universitario o de educación terciaria. Como se puede observar en la etapa correspondiente a la Gala de Ballet VI, las tres cuartas partes del público son mujeres. Un $50 \%$ de las mujeres que asisten al ballet se agrupa en la franja de edades entre 30 y 70 años en proporciones similares. Un importante porcentaje del público (18\%) tiene menos de 30 años, mientras que un $11 \%$ es mayor de 70 . Entre los varones la distribución es más pareja pues se ubica alrededor del $6 \%$ en cada grupo de edad. El nivel educativo en la población masculina también es elevado y predomina la educación terciaria.

El público de Romeo y Julieta tiene un perfil más joven que el de la Gala de Ballet VI. Se registra una leve disminución de los mayores de 70 años (8\%) y un significativo aumento de la franja de entre 30 y 49 años (35\%) y de los menores de 30 años (27\%).

La Gala del Ballet es un espectáculo que se presenta una vez al año y que, en lugar de tener una estructura narrativa unitaria clásica, se fragmenta en varios espectáculos de distintas expresiones de danza. La Gala $V I$, en la que se realizó la encuesta, presentó tres estrenos nacionales: «Theme and Variations», de George Balanchine, «Chacona», de Goyo Montero, y «Encuentros», de Marina Sánchez. Corresponde a la Gala 2017.

5 Agradecemos a Eliana Gonnet el diseño de los gráficos de este trabajo.

6 Está previsto llevar a cabo el estudio, comenzado en julio del 2017, a lo largo de un año de espectáculos.

7 Los grupos de edades fueron: menores de 30 años para englobar al público joven, mayores de 70 años para caracterizar al de edad avanzada, y las edades adultas se dividieron en adultos jóvenes (de 30 a 49 años) y adultos maduros (de 50 a 69 años). 
El promedio de edad es de 42 años. La relación hombre/mujer permanece prácticamente igual que para la Gala, al igual que el nivel educativo, que en las dos obras muestra un alto porcentaje de espectadores con estudios terciarios (65\%).

Cuadro 2. Distribución del público por edad, sexo y espectáculo

\begin{tabular}{|l|c|c|}
\hline & Gala & Romeo y Julieta \\
\hline Menor de 30 años & $24,4 \%$ & $27,0 \%$ \\
\hline De 30 a 49 años & $29,8 \%$ & $35,5 \%$ \\
\hline De 50 a 69 años & $30,2 \%$ & $29,6 \%$ \\
\hline De 70 años y más & $15,5 \%$ & $7,8 \%$ \\
\hline Total & $100 \%$ & $100 \%$ \\
\hline Promedio & 49 años & 42 años \\
\hline Hombre & $25,1 \%$ & $23,0 \%$ \\
\hline Mujer & $74,9 \%$ & $77,0 \%$ \\
\hline Total & $100 \%$ & $100 \%$ \\
\hline
\end{tabular}

Gráfico 1. Distribución por edades, Romeo y Julieta

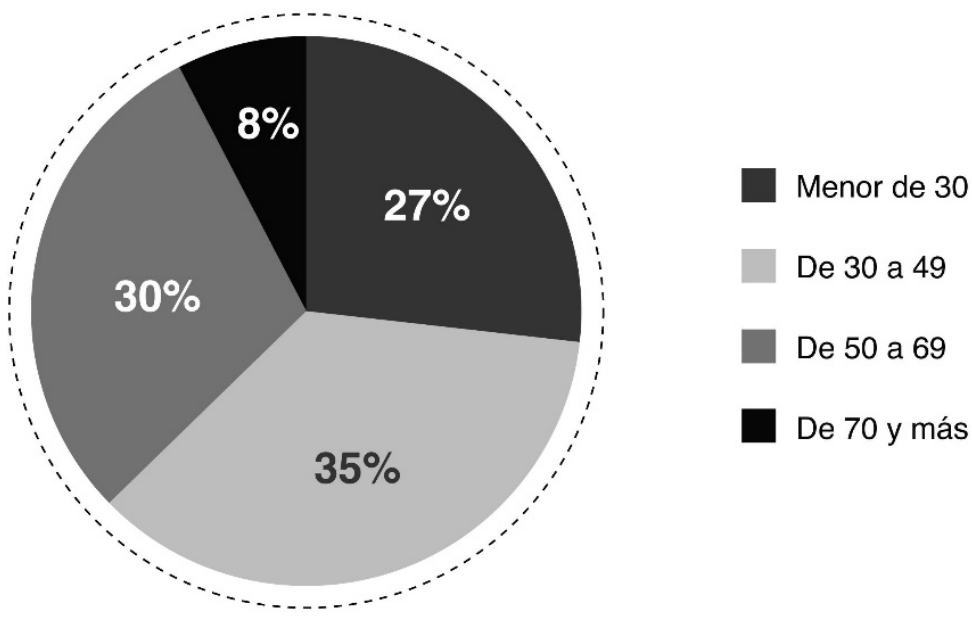


Gráfico 2. Distribución por nivel educativo, Romeo y Julieta

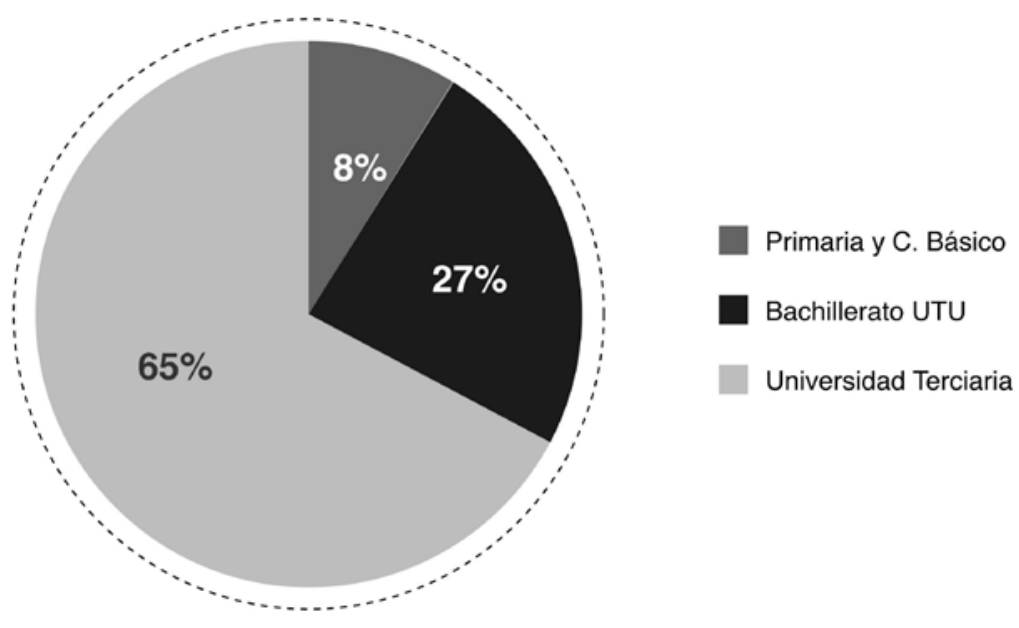

El público de la Gala es predominantemente montevideano. Un 88\% declara vivir en la capital del país, mientras que un $3 \%$ reside en el exterior y un $9 \%$ en el interior. Un $50 \%$ de los espectadores pertenece a barrios de nivel socioeconómico medio alto ubicados en la franja costera de la capital. Un $20 \%$ reside en la zona céntrica y los restantes, en porcentajes menores, se ubican en otros barrios. El público de Romeo y Julieta no presenta grandes variaciones con respecto a la medición anterior. Un $43 \%$ de quienes indicaron su lugar de residencia pertenece a barrios de nivel socioeconómico medio alto ubicados en la franja costera de Montevideo. Un 22\% vive en la zona céntrica. Los restantes porcentajes se concentran en la zona de Prado-Atahualpa (11\%) y La Blanqueada-Unión (9\%). 
Gráfico 3. Distribución del público por barrios de residencia. Gala de Ballet VI. En porcentajes

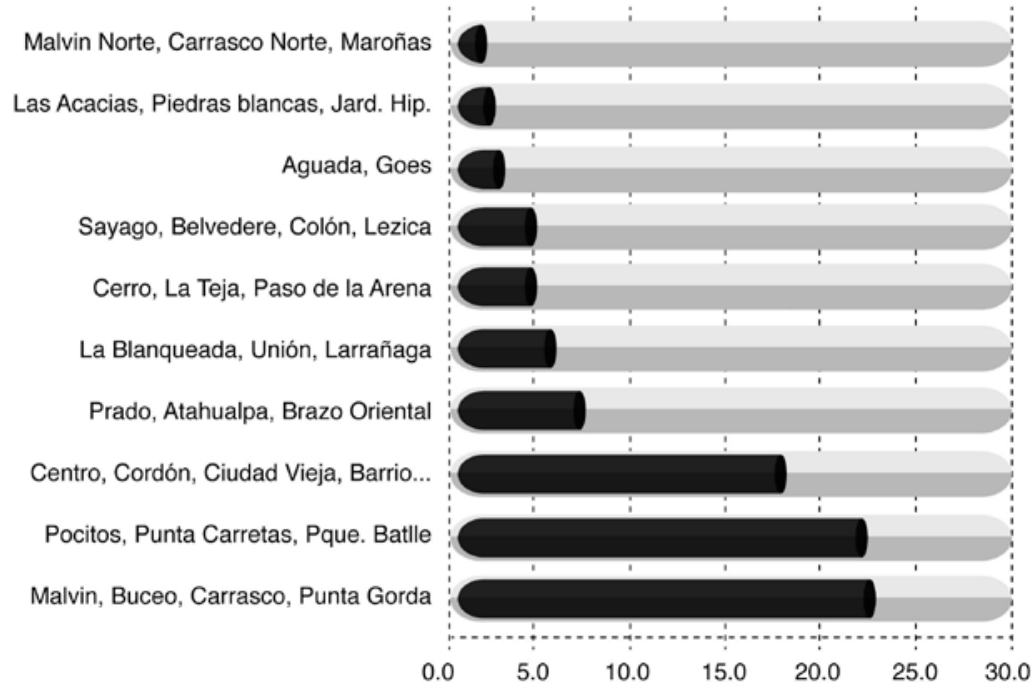

\section{Motivos y características de la asistencia de los espectadores}

De los espectadores de la Gala VI, un $11 \%$ asiste solo, un $30 \%$ asiste con un amigo o con un grupo de amigos, un $31 \%$ asiste con su pareja y un $27 \%$ con otros familiares. En Romeo y Julieta la proporción de personas que asisten solas desciende notoriamente: $3 \%$. La asistencia al ballet en familia también es importante, ya que un $31 \%$ de los espectadores concurre acompañado por un familiar. El ballet se perfila entonces como una actividad donde la interacción social se presenta como muy significativa. 
Gráfico 4. Con quién asiste al ballet. Gala de Ballet VI

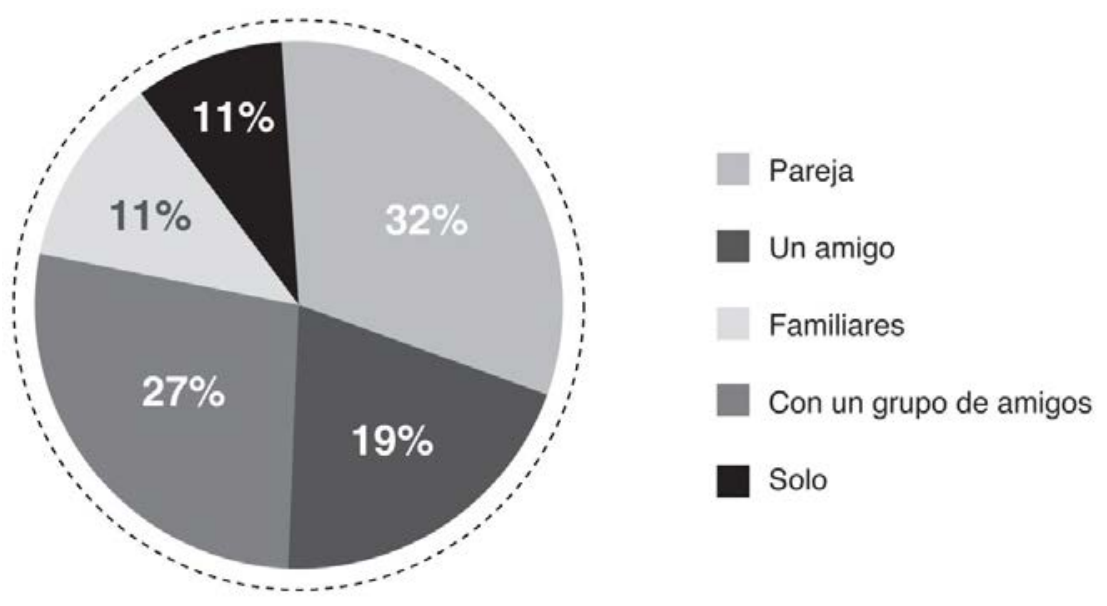

Gráfico 5. Con quién asiste al ballet. Romeo y Julieta

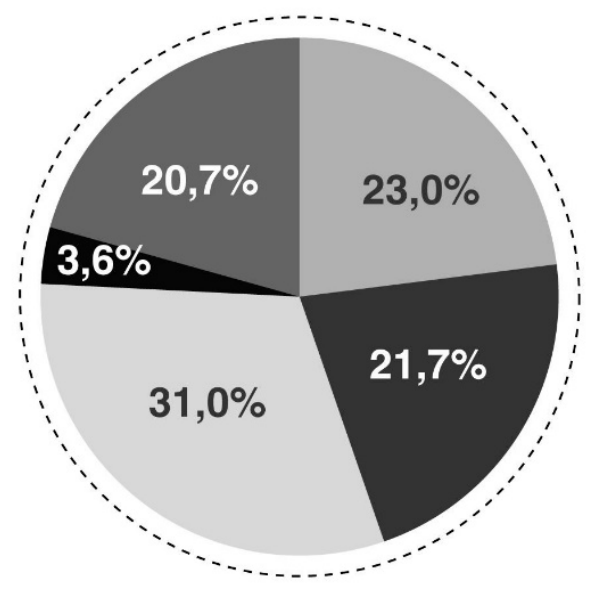

Pareja

Un amigo

Familiares

Con un grupo de amigos

$\square$ Solo 
El ballet no parece tener un público exclusivo, dado que los espectadores declaran asistir a otro tipo de espectáculos. Entre estos, el cine y el teatro registran un elevado nivel de asistencia, mientras que el carnaval y el fútbol, reconocidos como espectáculos populares, presentan un porcentaje menor.

Gráfico 6. Concurrencia a otros espectáculos. Gala de Ballet VI

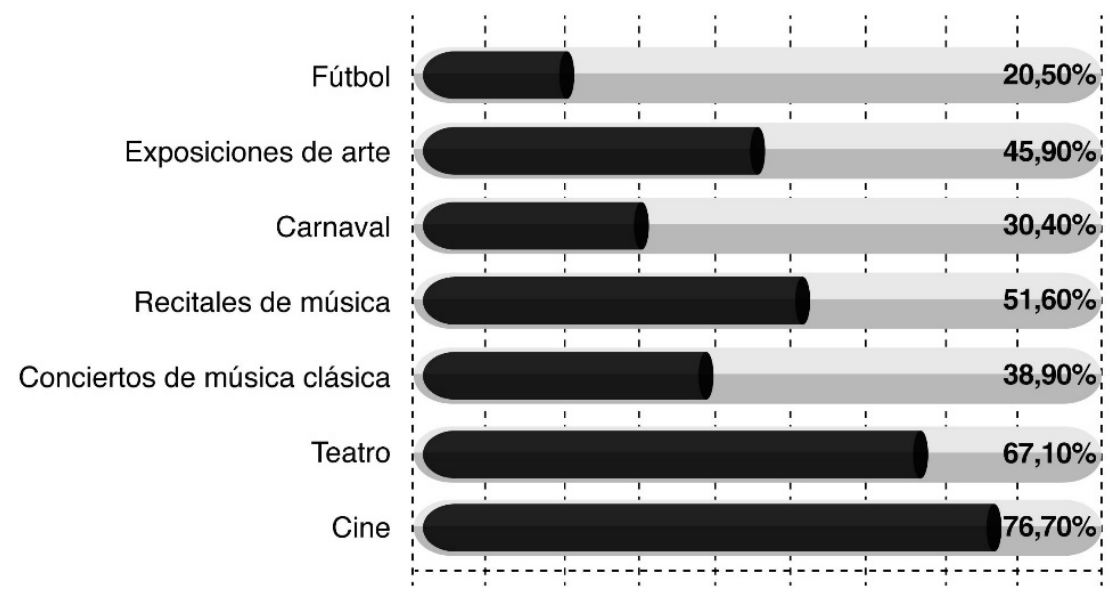

También se indagó acerca de las actividades artísticas que practica el público que asiste al ballet, y se encontró que la quinta parte de los espectadores declararon practicar algún tipo de danza. Entre un $15 \%$ y $17 \%$ toca un instrumento musical, en tanto otras actividades artísticas tienen menor relevancia. Un 57\% del público de Romeo y Julieta y un 39\% del de la Gala declararon no realizar ninguna actividad artística.

Gráfico 7. Prácticas artísticas en el tiempo libre. Romeo y Julieta 


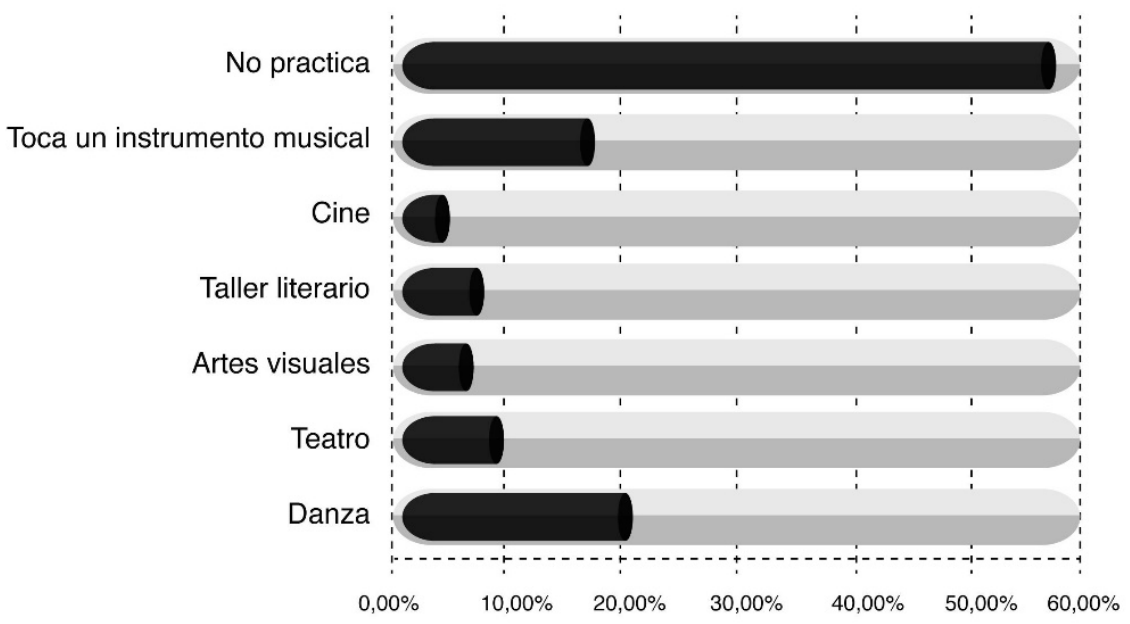

Casi una quinta parte de los espectadores asistieron por primera vez al ballet en la función de la Gala VI. Un 73\% de los asistentes a la Gala comenzaron a ir al ballet entre 2010 y 2017 . Aproximadamente un $26 \%$ fueron alguna vez antes de la llegada de Julio Bocca (2010). En el caso de Romeo y Julieta, más de una cuarta parte de los espectadores asistía por primera vez a una función del BNS.

Cudro 3. Asistencia por primera vez al BNS por espectáculo. En porcentaje

\begin{tabular}{|l|c|c|}
\hline & Gala & Romeo y Julieta \\
\hline No & $81,1 \%$ & $74,1 \%$ \\
\hline Sí & $18,9 \%$ & $25,9 \%$ \\
\hline Total & $100,0 \%$ & $100,0 \%$ \\
\hline
\end{tabular}

Cuadro 4. Año en que asistió por primera vez a un espectáculo del BNS - Gala de Ballet VI

\begin{tabular}{|l|c|}
\hline Período & Porcentaje de espectadores \\
\hline De 1950 a 2000 & $18,6 \%$ \\
\hline De 2000 a 2010 & $7,0 \%$ \\
\hline De 2010 a 2017 & $73,2 \%$ \\
\hline
\end{tabular}


La mayor parte de los espectadores son asistentes frecuentes al ballet, dado que van más de tres veces al año. Tanto entre aquellos consultados durante la Gala como en Romeo y Julieta, un $40 \%$ asiste entre dos y tres veces al año. Para Romeo y Julieta, casi una cuarta parte declaró asistir una vez por año. Este número coincide con el porcentaje de personas que declararon ver al BNS por primera vez. Este alto porcentaje en esta obra está probablemente ligado a las circunstancias particulares que se dieron a partir de la divulgación mediática del alejamiento de Julio Bocca (se presentaba como la última oportunidad de ver el «ballet de Bocca») y la incógnita sobre el futuro (aún no se había designado a su sucesor). También se podría afirmar que el público de la Gala es de perfil «más fiel» al ballet en general, en tanto Romeo y Julieta capta más espectadores emergentes.

Cuadro 5. ¿Con qué frecuencia asiste al Ballet?

\begin{tabular}{|l|c|c|}
\hline & Gala & Romeo y Julieta \\
\hline Una vez al año & $16,2 \%$ & $23,2 \%$ \\
\hline Dos veces al año & $16,2 \%$ & $25,8 \%$ \\
\hline Tres veces al año & $23,1 \%$ & $16,7 \%$ \\
\hline Más de tres veces al año & $44,4 \%$ & $34,3 \%$ \\
\hline Total & $100 \%$ & $100 \%$ \\
\hline
\end{tabular}

La razón de asistencia a la Gala de Ballet coincide con el perfil del espectador frecuente: un 36\% de los encuestados respondió que había ido al ballet porque lo hace siempre. A su vez, un 18\% mencionó que le habían recomendado especialmente esa obra y un $9 \%$ que tenía buena crítica, lo cual traza el perfil de un público que asistió atraído específicamente por esa función y un 9\% por un bailarín en particular. En Romeo y Julieta se agregaron al formulario dos opciones que pasaron a ser las más seleccionadas: «Porque le gusta el ballet» fue la opción de un 63\% y «Por interés cultural» la de un $35 \%$. Por lo tanto, la razón de asistencia al ballet sigue coincidiendo con el perfil del espectador frecuente. 
Cuadro 6. ¿Por qué decidió venir hoy al Ballet?

\begin{tabular}{|l|c|c|}
\hline & \multicolumn{1}{|l|}{ Gala } & Romeo y Julieta \\
\hline Porque le recomendaron especialmente la obra & $18,0 \%$ & $12,0 \%$ \\
\hline Por curiosidad & $12,0 \%$ & $6,2 \%$ \\
\hline Porque lo invitaron & $17,7 \%$ & $12,9 \%$ \\
\hline Porque la obra tenía buena crítica & $8,1 \% \%$ & $7,1 \%$ \\
\hline Porque bailaba un(a) bailarín(a) que le gusta & $8,8 \% \%$ & $9,1 \%$ \\
\hline Porque siempre viene & $36,0 \%$ & $12,9 \%$ \\
\hline Porque le regalaron la entrada & $0 \%$ & $4,2 \%$ \\
\hline Porque le gusta el ballet & & $63,1 \%$ \\
\hline Por interés cultural & & $35,6 \%$ \\
\hline
\end{tabular}

\section{Opiniones acerca de la función y del BNS en general}

El espectáculo es altamente estimado por los espectadores. En el formulario, tenían la posibilidad de evaluarlo de 1 (malo) a 5 (excelente). La valoración mayoritaria fue de excelente, seguida por muy bueno, y un mínimo porcentaje consideró que había sido regular o bueno. Esa alta apreciación del espectáculo en general se extiende a las distintas categorías que lo componen.

Gráfico 8. Valoración general del BNS. Gala de Ballet VI

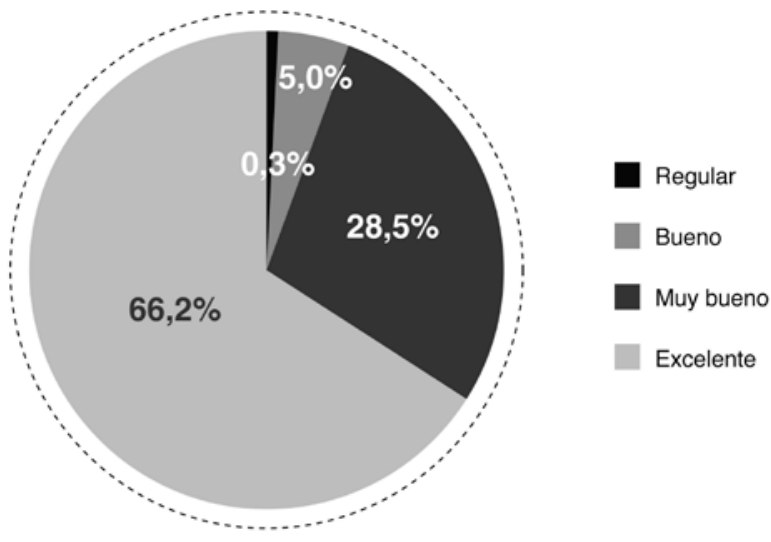


Gráfico 9. Valoración de la obra por rubro. Gala de Ballet VI

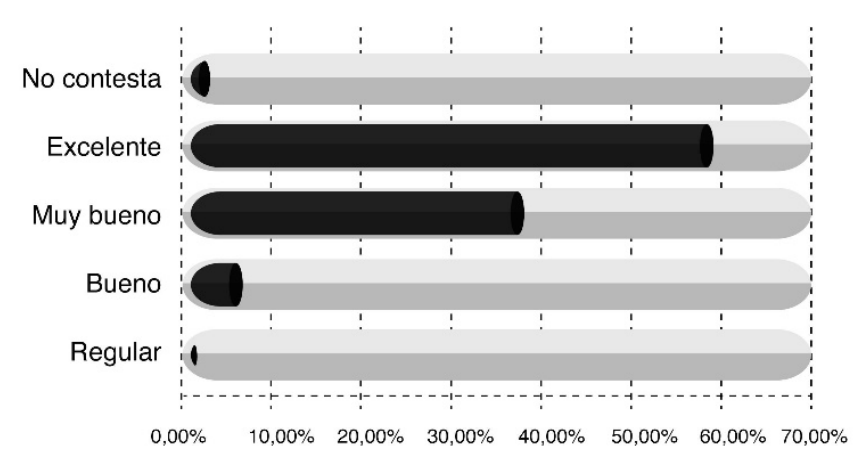

Gráfico 10. Valoración general del BNS. Romeo y Julieta

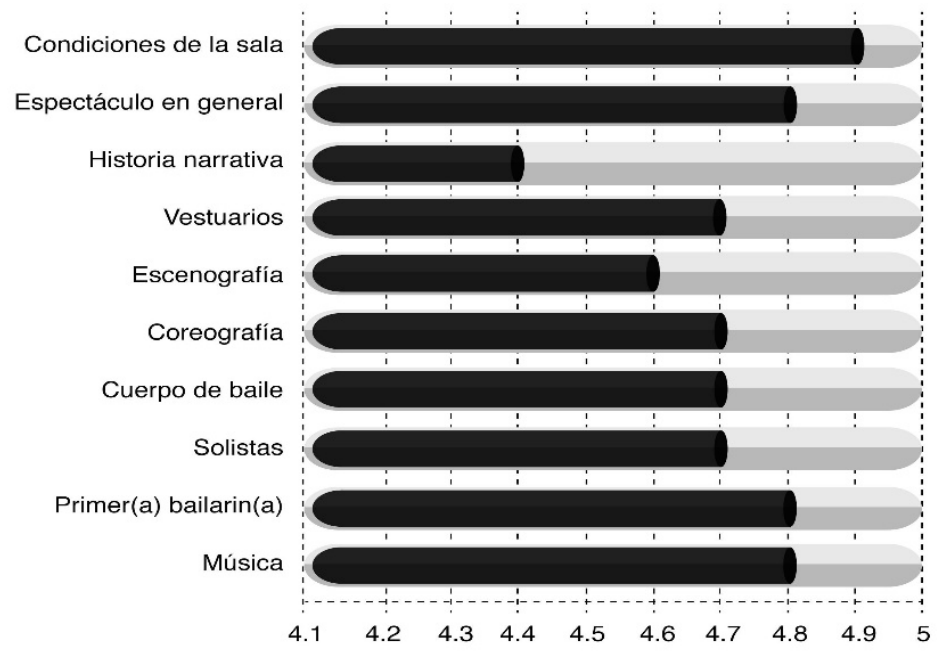

En cuanto a la valoración del nivel general del BNS por los espectadores de Romeo y Julieta, un 57,3\% lo considera excelente, un 36,2\% muy bueno y un mínimo porcentaje regular $(0,3 \%)$ o bueno $(4,9 \%)$. La valoración también es alta en las distintas categorías incluidas en el cuestionario, con porcentajes similares a la medición de la Gala de Ballet.

Esta valoración concreta sobre el espectáculo que los encuestados acaban de ver se complementa en el estudio con preguntas de carácter más abstracto, con las que se apunta a indagar sobre la apropiación simbólica que el público hace del ballet como 
práctica cultural y los imaginarios relacionados con esta práctica. La pregunta 9.1, por ejemplo, plantea al encuestado: ¿Se considera usted admirador del BNS?, mientras que la pregunta 18 inquiere: ¿Considera que el BNS está a la altura de las grandes compañías del mundo? Se trata de preguntas cerradas que el encuestado responde Sí, No o No sabe.

En las respuestas es elocuente la alta valoración de la compañía nacional en el imaginario del público. Un 71\% de los encuestados en la Gala de Ballet declara que el BNS está a la altura de las mejores compañías del mundo, mientras que únicamente un $3,9 \%$ no cree que esté entre las mejores y el restante $25,1 \%$ no lo sabe. Para los espectadores de Romeo y Julieta la valoración desciende levemente: un 61\% considera que el BNS está a la altura de las mejores compañías del mundo, mientras que un 3,6\% cree que no lo está.

Cuadro 7. ¿Considera que el BNS está a la altura de las grandes compañías del mundo?

\begin{tabular}{|l|c|c|}
\hline & Gala & Romeo y Julieta \\
\hline Sí & $71,0 \%$ & $61,2 \%$ \\
\hline No & $3,9 \%$ & $3,6 \%$ \\
\hline No sabe & $25,1 \%$ & $35,2 \%$ \\
\hline Total & $100 \%$ & $100 \%$ \\
\hline
\end{tabular}

Gráfico 11. ¿Se considera usted admirador del BNS? Romeo y Julieta

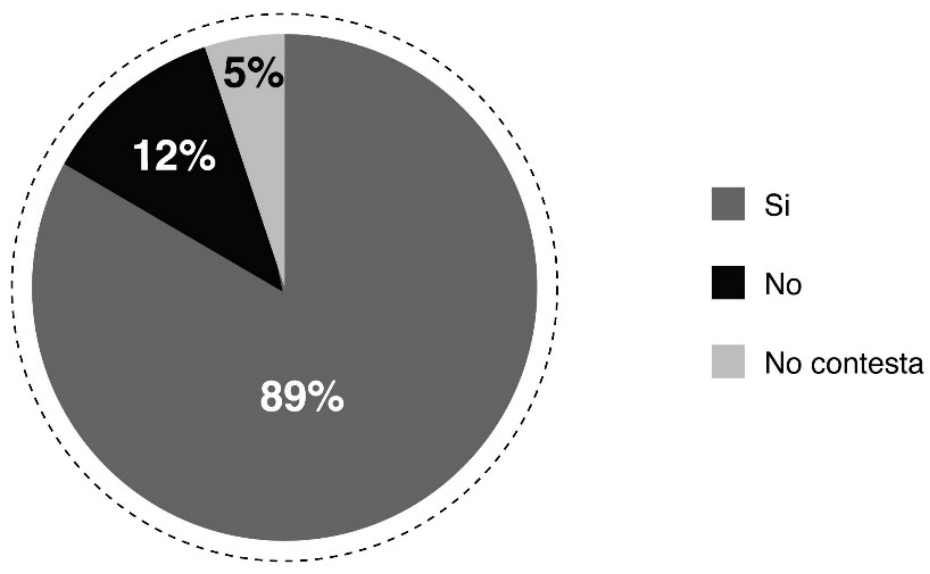


Como se advierte en la gráfica, el BNS ha generado un sentido de admiración importante en el público (un $83 \%$ de quienes vieron Romeo y Julieta se considera admirador del BNS). Por otra parte, la figura de Julio Bocca aparece fuertemente ligada a la imagen actual del BNS. Casi un $90 \%$ de los encuestados desconocen quién fue el anterior director artístico. El cruce de este dato con los de las edades de la población que asiste al ballet, así como con las fechas en que los espectadores declararon asistir por primera vez a un espectáculo del BNS, permite establecer a manera de hipótesis que con la refundación del BNS han surgido nuevos públicos en torno a una práctica cultural que durante años no generó una adhesión significativa.

\section{Conclusiones}

Debido al carácter preliminar de la información aquí presentada, y dado que la investigación se encuentra aún en curso, en lugar de conclusiones, es preferible plantear el análisis descriptivo de estos primeros resultados y esbozar algunas reflexiones con el fin de explorarlas en el futuro. En este sentido, surge que el público que asiste al Ballet Nacional del Sodre es predominantemente femenino, de edades adultas y de barrios costeros de la ciudad de nivel socioeconómico medio-alto. Aun así, se registran públicos emergentes que no responden a este perfil clásico del espectador de ballet: edades más jóvenes y barrios más periféricos. Este público está más presente en la función de Romeo y Julieta, donde una proporción mayor asiste por primera vez al espectáculo. En la Gala del Ballet es más frecuente el público clásico; no obstante, la mayor parte de este público empezó a ver ballet a partir de 2010, lo cual define la captación de nuevos espectadores por la compañía en la última década.

La forma de asistir al BNS es predominantemente familiar; más de la mitad del público va con su pareja y/o con otros familiares. Cabe pensar también que probablemente sea una actividad intergeneracional, en la que madres y abuelas transmiten a hijas y nietas el gusto por esta clase de espectáculo. Los espectadores asisten también a espectáculos de otro tipo: cine, teatro, exposiciones de arte y recitales de música, lo que indica que el Ballet no capta en exclusividad a un público poco expuesto, sino que empieza a compartir el mercado de ofertas culturales con espectáculos diversos. De todas formas, se puede afirmar que los espectadores del ballet son un público frecuente que asiste más de una vez al año, básicamente porque le gusta y porque tiene costumbre de asistir.

Las opiniones del público sobre la compañía son muy favorables: una gran mayoría califica como muy bueno o excelente el espectáculo al que acaba de asistir y elogia varios rubros, como la música, los bailarines y el vestuario, además de manifestar una alta valoración por las condiciones de la sala. Entre un 60 y un $70 \%$ de los espectadores 
consideran que el Ballet Nacional del Sodre se encuentra a la altura de las grandes compañías del mundo y un $83 \%$ se considera admirador de este cuerpo de baile. Si bien de manera preliminar se constata la relevancia que tuvo la figura de Julio Bocca en la consolidación de la compañía, resta explorar su valoración en el futuro, con mayor profundidad, a través de técnicas cualitativas.

Por otro lado, entre los asistentes se distinguen algunos subgrupos bien diferenciados que permiten establecer, a modo de hipótesis, una tipología del público del Ballet conformada por tres categorías:

- público histórico,

- público contemporáneo,

- nuevos públicos.

El público histórico está conformado por aquellos espectadores (espectadoras en su mayoría) de mayor edad que asisten asiduamente al ballet desde antes de que Julio Bocca se hiciera cargo de la dirección artística. Es entonces el público más fiel, que ha permanecido cercano a esa práctica cultural y que ha podido seguir las transformaciones que el BNS experimentó en los últimos años. Se trata, por lo tanto, a los efectos de esta investigación, de un público clave para entender y conocer de primera mano cómo se han experimentado estos cambios desde su punto de vista.

El segundo subgrupo está conformado lo que denominamos público contemporáneo. Ubicamos en esta categoría a aquellos espectadores de todas las edades que comenzaron a asistir a los espectáculos del BNS a partir de 2010, con la llegada de Julio Bocca, y que van asiduamente (dos o tres veces por año) a los espectáculos que la compañía estrena. Se trata de un público en general más joven que los espectadores de siempre y que no conoció las etapas anteriores del BNS. A la vez, es un público contemporáneo que ha desarrollado una fidelidad con la compañía, algo que se demuestra en la frecuencia con la que asiste desde que comenzó con esta práctica cultural, y en un alto porcentaje se declara admirador del BNS.

Al tercer subgrupo lo denominamos nuevos públicos. Se compone de grupos etarios diversos, mayoritariamente jóvenes, que cuando fueron encuestados habían ido por primera vez a un espectáculo de ballet. El porcentaje de los que asistían por primera es muy alto tanto en la Gala VI como en Romeo y Julieta. Por lo tanto, se trata de un público que recién comienza a ser objeto de análisis, tanto desde el punto de vista sociodemográfico como de sus motivaciones y representaciones acerca de esta práctica cultural. Estos nuevos públicos permiten pensar que el Ballet Nacional aún se encuentra en una etapa de crecimiento institucional desde su refundación. 
La aproximación y el conocimiento que puede proveer un estudio de públicos permiten recabar la opinión de los espectadores y su valoración del espectáculo, así como conocer su perfil sociodemográfico, sus prácticas habituales y su vínculo con otras formas de expresión cultural. En el caso del BNS, las transformaciones que ha tenido la compañía en los últimos años son valoradas por aquellos que asisten habitualmente a sus espectáculos. En un año de cambios en la dirección artística, con la llegada del maestro Igor Yebra en 2018, un estudio de estas características se presenta como una herramienta pertinente para evaluar la percepción de ese público. Eventualmente, la incorporación sistemática de este tipo de información sobre públicos podría contribuir a una mayor profesionalización de la gestión, aspecto sin duda de interés para quienes están al frente de la toma de decisiones en políticas culturales.

\section{Referencias bibliográficas}

ACHugar, H.; RAPETTI, S.; Dominzain, S. y RADAKovich, R. (2002). Imaginarios y consumo cultural: Primer informe nacional sobre consumo y comportamiento cultural. Uruguay, 2002. Montevideo: FHCE.

AUditorio NACIONAL DEL SODRE (s/f). «Giselle se consagra como la obra más exitosa del BNS». Recuperado de http://www.auditorio.com.uy/uc_768_1.html.

AVIONES DE PAPEL; PROARTES (2016). Investigación cualitativa de públicos de danza contemporánea. Recuperado de http://www.danzacostarica.info/.

Bourdieu, P. y DARBEL, A. (2003). L'amour de l'art. París: Les Éditions de Minuit.

CAMPOS, L. (2014). Luces y sombras del Ballet Nacional del SODRE. (Memoria de grado).

Universidad Católica del Uruguay, Montevideo.

CLAEH (2010). Efecto Cine: 2008-2010. Montevideo: CLAEH.

Coulangeon, P. (2005). Sociologie des pratiques culturelles. París: La Découverte.

Craine, D. y Mackrell, J. (2010). Oxford dictionary of dance. Oxford: Oxford University Press.

Dominzain, S.; RAdakovich, R.; DuARTE, D. y CASTElli Rodríguez, L. (2014). Imaginarios y consumo cultural: Tercer informe nacional sobre consumo y comportamiento cultural. Montevideo: FHCE.

Ministerio de Cultura de España. Laboratorio Permanente de Público de Museos (2011). Conociendo a nuestros visitantes: Estudio de público en museos del Ministerio de Cultura. Recuperado de http://es.calameo.com/read/0000753353c6f6cc139ef.

URRUTIAGUER, D. (2014). «Les représentations du public dans le monde de la danse contemporaine». Quaderni, 83, 29-40. 
Wortman, A. y De Angelis, C. (2017). El dinero es lo de menos: A propósito del público del festival BAFICI. Recuperado de https://www.academia.edu/32964348/ el_dinero_es_lo_de_menos._a_prop\%c3\%b3sito_del_p\%c3\%bablico_del_festival_bafici.

WORTMAN, A. (2015). «De la americanización a la globalización de los espectadores». Versión. Estudios de comunicación y política, 36, 113-125.

Wortman, A.; Bayardo, R. (2012). «Consumos culturales en Argentina». Alteridades, 22, 11-21. 